\title{
Age-related clonal hematopoiesis and monoclonal B-cell lymphocytosis / chronic lymphocytic leukemia: a new association?
}

\section{Adalgisa Condoluci and Davide Rossi}

Hematology, Institute of Oncology Research, and Oncology Institute of Southern Switzerland, Bellinzona, Switzerland

E-mail: davide.rossi@eoc.ch

doi:10.3324/haematol.2018.191098

A gathangelidis et al. ${ }^{1}$ compared the mutational landscape of low-count monoclonal B-cell lymphocytosis (MBL), high-count MBL and highly stable chronic lymphocytic leukemia (CLL) with confirmed lack of progression after a very long follow up (>10 years). The Authors also studied the polymorphonuclear (PMN) fraction and germline DNA from buccal swabs of the same individuals. Whole genome sequencing was complemented with deep sequencing of targeted genes.

The sample size, along with the low coverage imposed by whole genome sequencing, are both limitations in efforts to discover yet unknown variants that might actually be recurrent in these conditions. While Agathangelidis et al. acknowledge these limitations, three major findings characterize their manuscript. ${ }^{1}$ First, lowcount $\mathrm{MBL}$, high-count $\mathrm{MBL}$ and highly stable CLL share a similar genetic landscape and mutational signatures, which include the presence of mutations in known drivers associated with poor outcome, such as NOTCH1, $S F 3 B 1, P O T 1,{ }^{2-4}$ indicating that these mutations are not sufficient to drive the aggressiveness of the disease by themselves. Second, the mutational landscape of paired PMN suggests that most of these patients carry a clonal hematopoiesis that could possibly be age-related. Third, a number of somatic mutations were found in both the MBL/CLL cells and PMN, supporting the idea that the $\mathrm{MBL} / \mathrm{CLL}$ clone stemmed from a common ancestral hematopoietic precursor that was able to participate in both lymphoid and myeloid differentiation. By documenting that the DNA from PMN was free from contamination by MBL/CLL DNA, for example, by using molecular minimal residual disease methods relying on the individual patient's specific immunoglobulin gene rearrangements, the Authors have provided further evidence of this important finding which, although previously reported, ${ }^{5,6}$ had remained a subject of debate.

Several hematologic malignancies, including CLL, multiple myeloma (MM) and acute myeloid leukemia (AML), have well-defined precursor states that precede the development of overt cancer. CLL is always preceded by a high $\mathrm{MBL}$ count, ${ }^{7} \mathrm{MM}$ is almost always preceded by monoclonal gammopathy of undetermined significance (MGUS), ${ }^{8}$ and at least a quarter of all patients with myelodysplastic syndromes (MDS) have disease that evolves into AML. ${ }^{9}$ Deep genomic sequencing of normal subjects revealed that during human aging, the expansion of 1 or more hematopoietic stem and progenitor cells (HSPC) will result in clones that will sustainably contribute more than others to the production of mature blood cells. Accordingly, age-related clonal hematopoiesis (ARCH) is defined as the expansion of HSPC clones, harboring specific, disruptive, and recurrent genetic variants, in individuals without clear diagnosis of hematologic malignancies. ${ }^{10} \mathrm{MDS}$ are frequently preceded by $\mathrm{ARCH} .{ }^{11}$

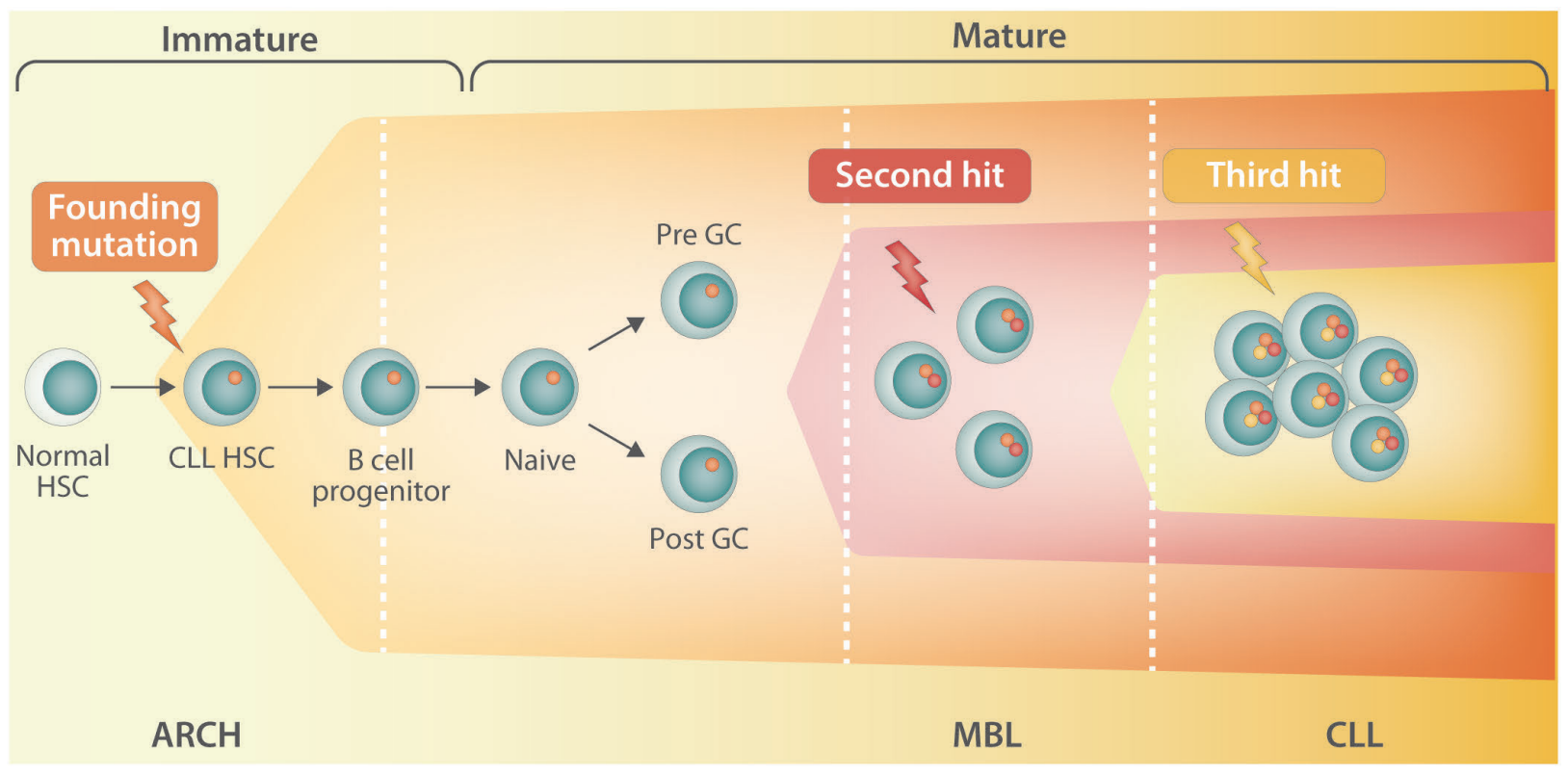

Figure 1. Hypothetical model of evolution from age-related clonal hematopoiesis to monoclonal B-cell lymphocytosis/chronic lymphocytic leukemia. 
Some ARCH-related mutations can increase the risk for leukemia, ${ }^{12}$ while others possibly increase the risk for heart disease and diabetes. ${ }^{13}$ From a pathogenetic standpoint, the study of Agathangelidis et al. ${ }^{1}$ provides the proof of principle that ARCH may also associate with expansion of B-cell clones with CLL phenotype, and connects $\mathrm{ARCH}$ with MBL and CLL in a continuum of evolution from HSCP clones to mature B-cell clones (Figure 1), thus validating in vivo in patients the notion initially reported from mice studies that the propensity to generate clonal B cells has already been acquired at the HSCP stage. ${ }^{14}$ To robustly establish this association and to gain greater insight into the pathogenetics, larger cohorts of MBL and CLL patients should be investigated with the rigorous approach utilized by Agathangelidis et al. ${ }^{1}$

One of the long-term complications of chemoimmunotherapy in CLL is the development of treatmentrelated MDS/AML. ${ }^{15}$ Chemoimmunotherapy poses a strong selection bottleneck to HSCPs, and thus only the fittest HSCPs survive and repopulate after the stress of chemoimmunotherapy. ${ }^{16}$ HSCP fitness may be sustained by somatic mutations in the context of a preceding $\mathrm{ARCH}$, and it is increasingly recognized as a risk factor for therapy-related MDS/AML. ${ }^{17}$ Among elderly patients who receive chemotherapy and develop therapy-related MDS/AML, most have ARCH before chemotherapy. Consistently, $\mathrm{ARCH}$ associates with an increased rate of therapy-related AML/MDS. ${ }^{17}$ Following this line of evidence, the study by Agathangelidis et al. ${ }^{1}$ prompts investigation into whether the finding of an $\mathrm{ARCH}$ in CLL patients who receive chemoimmunotherapy is a risk factor for the development of therapy-related MDS/AML. If this is proved to be the case, given the availability of novel agents for the treatment of CLL that are not stressful for HSCP, ARCH might become a new biomarker for tailoring treatment in CLL.

\section{References}

1. Agathangelidis A, Ljungström V, Scarfò L, et al. Highly similar genomic landscapes in monoclonal B-cell lymphocytosis and ultrastable chronic lymphocytic leukemia with low frequency of driver mutations. Haematologica. 2018;103(5):865-873.

2. Rossi D, Rasi S, Fabbri G, et al. Mutations of NOTCH1 are an independent predictor of survival in chronic lymphocytic leukemia. Blood. 2012;119(2):521-529.

3. Wang L, Lawrence MS, Wan Y, et al. SF3B1 and other novel cancer genes in chronic lymphocytic leukemia. $N$ Engl J Med. 2011;365(26):2497-2506.

4. Herling CD, Klaumünzer M, Rocha CK, et al. Complex karyotypes and KRAS and POT1 mutations impact outcome in CLL after chlorambucil-based chemotherapy or chemoimmunotherapy. Blood. 2016;128(3):395-404

5. Damm F, Mylonas E, Cosson A, et al. Acquired initiating mutations in early hematopoietic cells of CLL patients. Cancer Discov. 2014;4(9):1088-1101.

6. Quijada-Álamo M, Hernández-Sánchez M, Robledo C, et al. Nextgeneration sequencing and FISH studies reveal the appearance of gene mutations and chromosomal abnormalities in hematopoietic progenitors in chronic lymphocytic leukemia. J Hematol Oncol. 2017;10(1):83.

7. Landgren $\mathrm{O}$, Albitar M, Ma W, et al. B-cell clones as early markers for chronic lymphocytic leukemia. N Engl J Med. 2009;360(7):659667

8. Landgren O, Kyle RA, Pfeiffer RM, et al. Monoclonal gammopathy of undetermined significance (MGUS) consistently precedes multiple myeloma: a prospective study. Blood. 2009;113(22):5412-5417.

9. Pfeilstöcker M, Tuechler H, Sanz G, et al. Time-dependent changes in mortality and transformation risk in MDS. Blood. 2016;128(7):902-910.

10. Jaiswal S, Fontanillas P, Flannick J, et al. Age-related clonal hematopoiesis associated with adverse outcomes. N Engl J Med. 2014:371(26):2488-2498.

11. Malcovati L, Gallì A, Travaglino E, et al. Clinical significance of somatic mutation in unexplained blood cytopenia. Blood. 2017;129(25):3371-3378.

12. Genovese G, K"ahler AK, Handsaker RE, et al. Clonal hematopoiesis and blood-cancer risk inferred from blood DNA sequence. $\mathrm{N}$ Engl J Med. 2014;371(26):2477-2487.

13. Jaiswal S, Natarajan P, Silver AJ, et al. Clonal hematopoiesis and risk of atherosclerotic cardiovascular disease. $N$ Engl J Med. 2017;377(2):111-121.

14. Kikushige Y, Ishikawa F, Miyamoto $\mathrm{T}$, et al. Self-renewing hematopoietic stem cell is the primary target in pathogenesis of human chronic lymphocytic leukemia. Cancer Cell. 2011;20(2):246259

15. Benjamini O, Jain P, Trinh L, et al. Second cancers in patients with chronic lymphocytic leukemia who received frontline fludarabine, cyclophosphamide and rituximab therapy: distribution and clinical outcomes. Leuk Lymphoma. 2015;56(6):1643-1650.

16. Wong TN, Ramsingh G, Young AL, et al. Role of TP53 mutations in the origin and evolution of therapy-related acute myeloid leukaemia. Nature. 2015;518(7540):552-555

17. Takahashi K, Wang F, Kantarjian H, et al. Preleukaemic clonal haemopoiesis and risk of therapy-related myeloid neoplasms: a casecontrol study. Lancet Oncol. 2017;18(1):100-111. 